TRANSACTIONS OF THE

AMERICAN MATHEMATICAL SOCIETY

Volume 183, September 1973

\title{
REMARKS ON GLOBAL HYPOELLIPTICITY
}

BY

\author{
STE PHEN J. GREENF IE LD(1) AND NOLAN R. WALLACH( ${ }^{2}$ )
}

ABSTRACT. We study differential operators $D$ which commute with a fixed normal elliptic operator $E$ on a compact manifold $M$. We use eigenfunction ex pansions relative to $E$ to obta in simple conditions giving global hypoellipicity. These conditions are equivalent to $D$ having parametrices in certain saces of functions or distributions. An example is given by $M=$ compact Lie group and and $E=$ Casimir operator, with $D$ any invariant differential operator. The connections with global subelliptic estimates are investigated.

0 . Introduction. We say a differential operator $D$ on a manifold $M$ is globally bypoelliptic $(\mathrm{GH})$ if when $D f=g$ (with $f \in \mathcal{D}^{\prime}(M), g \in C^{\infty}(M)$ ) then $f \epsilon$ $C^{\infty}(M)$. We begin this paper by recalling some Fourier analysis relative to an elliptic operator $E$ on a compact manifold $M$, and apply this to obtain simple conditions on the rate of growth of the Fourier transform (relative to $E$ ) of $D$ which are equivalent to $(\mathrm{GH})$. The growth conditions are interpreted as global solvability conditions.

We apply these theorems to the case: $M=$ compact homogeneous space, $E=$ invariant Laplace-Beltrami operator, and $D=$ any invariant differential operator. Some new examples are discussed. We note that global hypoellipticity seems to be quite directly connected with questions of number theory-unlike the (analytically) more delicate questions of local hypoellipticity. We connect the eigenfunction estimates of $E$-Fourier analys is with global subelliptic estimates.

Almost every result in this paper can be extended to differential operators on vector bundles (see Wallach [11] for the basic ideas).

We thank Carl Hoel and William Sweeney for patiently teaching us about differential equations. We also thank Richard Bumby for suggesting the use of Pell's equation in $\$ 3$.

1. Fourier analys is relative to an elliptic operator. Let $M$ be a compact manifold without boundary of dimension $n$ with a fixed volume element $d v$. Let $E$ be an elliptic, normal $\left(E E^{*}=E^{*} E\right)$ differential operator of order $e$ on $M$. We

Received by the editors July 10, 1972.

AMS (MOS) subject classifications (1970). Primary 35H05; Secondary 43A80.

(1) Partially supported by NSF GP-20647.

(2) Partially supported by Alfred P. Sloan Fellowship. 
review some basic results on Fourier analysis relative to $E$. Most of the statements here can be found in papers of Seeley ([8], [9]) or are easily derived from work there. We shall assume that $M, d v$, and $E$ are either $C^{\infty}$ or real analytic.

The eigenvalues of $E$ are a sequence $\left\{\lambda_{j}\right\}$, with $\lambda_{j} \neq \lambda_{k}$ when $j \neq k$. Each $\lambda_{j}$ corresponds to a finite dimensional eigenspace $F_{j}$ of $C^{\infty}$ (real analytic) functions on $M$. We put $d_{j}=\operatorname{dim} F_{j}$. We take $F_{0}=\operatorname{ker} E$, and $\lambda_{0}=0$. Note that $\left\{\lambda_{j}\right\}$ is subject to the restriction

$$
\sum_{j>0} d_{j}\left|\lambda_{j}\right|^{-2 n}<\infty
$$

We now analyze $C^{\infty}$ functions and distributions relative to $E . L^{2}(M)$ is the direct sum of the $F_{j}(j \geq 0)$. On each $F_{j}$ we put the induced Hilbert space structure. (Here, and in much that follows, the actual computational details depend greatly on the choice of $d v$.) Let $P_{j}: L^{2}(M) \rightarrow F_{j}$ be the corresponding projection.

Suppose $T \in \mathscr{D}^{\prime}(M)$ (the distributions on $M$ ). Let $j$ be a nonnegative integer. 1. Definition. $\hat{T}(j) \in F_{j}^{*}$ is defined by $\hat{T}(j)(f)=T f$, for $f \in F_{j}$.

$\hat{T}$ generalizes various notions of discrete Fourier transform. See $\$ 3$.

2. Theorem. Each $\hat{T}(j) \circ P_{j}$ is in $\mathscr{D}^{\prime}(M)$, and

$$
T=\sum_{j \geq 0} \hat{T}(j) \circ P_{j} \quad \text { in } \mathcal{D}^{\prime}(M)
$$

3. Theorem. There is $T \in \mathscr{D}^{\prime}(M)$ with $\hat{T}=S$ if and only if $\|S(j)\| \leq$ $K\left(\left|\lambda_{j}\right|+1\right)^{K}$ for some $K>0$ (i.e. $\|S(j)\|$ is of polynomial growtb).

We can define $H^{s}$ spaces relative to $E$.

4. Definition. $H_{E}^{s}(M)=$ f formal Fourier series $S$ with $S(j) \in F_{j}^{*}$ so that $\left.\Sigma_{j>0}\left|\lambda_{j}\right|^{2 s}\|S(j)\|^{2}<\infty\right\}$.

$H_{E}^{s}(M)$ is a Hilbert space with the obvious inner product. And $H_{E}^{s}(M)=$ $H^{s e}(M)$, where the latter is the usual Sobolev space on $M$.

We realize the $C^{\infty}$ functions as distributions using $d v$ : if $f \in C^{\infty}(M)$, put $f(g)=\int_{M} f g d v$, for all $g \in C^{\infty}(M)$. Then there are simple criteria in terms of the $E$-Fourier series for an element of $D^{\prime}(M)\left(=\bigcup H_{E}^{s}(M)\right.$ by Theorem 3$)$ to be in $C^{\infty}(M)$.

5. Theorem. $T \in \mathfrak{D}^{\prime}(M)$ is in $C^{\infty}(M)$ iff

$$
\sum_{j>0}\|\hat{T}(j)\|^{2}\left|\lambda_{j}\right|^{K}<\infty \quad \text { for every } K \quad \text { iff } \sup _{j>0} \frac{\|\hat{T}(j)\|}{\left|\lambda_{j}\right|^{K}}<\infty \quad \text { for every } K .
$$


In the real analytic case, we can give a similar recipe for real analytic functions, the set $A(M)$.

6. Theorem. $T \in \mathfrak{D}^{\prime}(M)$ is in $A(M)$ iff

$$
\sum_{j>0}\|\hat{T}(j)\|^{2 s} \mu_{j}<\infty \quad \text { for some } s>1 \text { iff } \sup _{j>p}\|\hat{T}(j)\|^{s} \mu_{j}<\infty \text { for some } s>1
$$

(where $\left.\mu_{j}=\left(\left|\lambda_{j}\right|\right)^{1 / e}, e=\operatorname{deg} E\right)$.

The continuous linear forms on $A(M)$ are the analytic functionals, $A^{\prime}(M)$. We characterize them simply:

7. Theorem. An E-Fourier series $S$ is an element of $A^{\prime}(M)$ iff $\Sigma\|S(j)\|^{2 t} \mu_{j}$ $<+\infty$, for all $t$ between 0 and 1. ( $\mu_{j}$ as in Theorem 6.)

8. Remark. The characterizations of Theorems 3, 5, and 6 are classical for (multiple) Fourier series on the : $n$-torus, $T^{n}$ (see Schwartz [7]).

2. Global hypoetlipticity for operators commuting with an elliptic operator. We continue the assumptions of $\S_{1}$ on $M, d v$, and $E$.

1. Definition. A differential operator $P$ on $M$ is $E$-invariant iff $E P=P E$.

If $P$ is $E$-invariant, $P\left(F_{j}\right) \subset F_{j}$. In the remainder of this section we assume that $P$ is $E$-invariant.

2. Definition. $\hat{P}(j)$ is the linear endomorphism of $F_{j}$ given by $P$.

We note that, in general, there may be no elliptic operator commut ing with a given operator $P$. (If there were, then $P$ would have discrete spectrum.)

We need to measure the rate of growth of $\hat{P}(j)$ as $j \rightarrow \infty$. We introduce two measurements .

3. Definition. Let $L: V \rightarrow W$ be a linear map between normed vector spaces. $M(L)=\sup \{\|L(v)\| ;\|v\|=1\}$, and $m(L)=\inf \{\|L(v)\| ;\|v\|=1\}$.

4. Remarks. Of course, $m$ and $M$ measure how much $L$ deforms the unit sphere. If $L$ is invertible, $M\left(L^{-1}\right)=(m(L))^{-1}$. If $V=W, \operatorname{dim} V<\infty$, then $L$ is invertible iff $m(L) \neq 0$.

5. Definition. $P$ is globally hypoelliptic (GH) iff whenever $P f=g, f \epsilon$ $D^{\prime}(M), g \in C^{\infty}(M)$, then $f \in C^{\infty}(M)$.

6. Theorem. $P$ is $(G H)$ if and only if there are positive real numbers $L, M$ so that

$$
m(\hat{P}(j)) \geq L\left|\lambda_{j}\right|^{M / e}
$$

for j sufficiently large. 
Proof. Suppose $P f=g$, with $f, g \in \mathscr{D}^{\prime}(M)$. Then $\hat{P}(j) \hat{f}(j)=\hat{g}(j)$.

$(\mathrm{LM}) \Rightarrow(\mathrm{GH})$. By (LM), $m(\hat{P}(j)) \neq 0$ if $j$ is large. So $\hat{P}(j)$ is invertible and $\hat{f}(j)=(\hat{P}(j))^{-1} \hat{g}(j)$ for $j$ large. (LM) becomes an estimate controlling the growth of $\hat{f}$ in terms of the growth of $\hat{g}$ and $M\left(\hat{P}^{-1}\right)=m(\hat{P})^{-1}$. Theorem 1.5 applies to show if $g \in C^{\infty}(M)$, then $f \in C^{\infty}(M)$.

$(\mathrm{GH}) \Rightarrow(\mathrm{LM})$. Suppose $(\mathrm{LM})$ is false. Then there is a sequence $\left\{f_{a}\right\} \epsilon$ $C^{\infty}(M)$ with $f_{a} \in F_{j a},\left\|f_{a}\right\|=1$, and $\left\|\hat{P}\left(j_{a}\right) f_{a}\right\|<1 /\left|\lambda_{j a}\right|^{j}$ (as $\left.\alpha \rightarrow \infty, j_{a} \rightarrow \infty\right)$. Consider $f=\Sigma f_{a}$. Using Theorems 1.3 and 1.5 we see easily that $f \in \mathscr{D}^{\prime}(M)-$ $C^{\infty}(M)$, and $P f \in C^{\infty}(M)$. So $(G H)$ is false. (Other versions of this proof are used in [1], [2], and [3].)

7. Remarks. If $P$ has order $N$, then $M(\hat{P}(j)) \leq C\left(\left|\lambda_{j}\right|\right)^{N / e}$. If $P$ is elliptic, Garding's inequality implies that we can take $M=N$ in (LM). (These are the reasons we put $M / e$ rather than $M$ in condition (LM). Also, the same $M$ will do if we consider $E^{p}$ Fourier series, $p$ an integer. See Remark 1.8.)

We define $h(p)=$ exponent of bypoellipticity of $P$ to be the supremum of the $M$ for which (LM) holds. Put $b(P)=-\infty$ if (LM) is false for all $M$. Then $h(P)$ $\leq N$. Note that $b(P)$ can vary erratically with the coefficients of $P$ if $P$ does not have any of the more gross properties of hypoellipticity. (See the first order examples in [1], [2], and [3]. Also see the appendix.)

The estimate (LM) can be interpreted in the context of Sobolev spaces as a simple "subelliptic estimate". We discuss this in the appendix.

We consider adjoints of $E$-invariant (GH) operators, using the following simple lemma.

8. Lemma. Let $W$ be a finite dimensional Hilbert space, and $T: W \rightarrow W a$ linear map. Then $m(T)=m\left(T^{*}\right)$.

$d v$ identifies $F_{j}$ and $F_{j}^{*}$, so that $\widehat{P^{*}}(j)=\hat{P}(j)^{*} . P^{*}$ is the $L^{2}$-adjoint differential operator of $P$. Then Lemma 8 and The orem 6 immediately give the following result.

9. Theorem. If $P$ is (GH), then so is $P^{*}$.

Note that there are hypoelliptic operators whose adjoints are not hypoelliptic.

The condition (LM) also gives information on solvability. The results below can be considered (in view of Theorem 9) as very simple examples of the "hypoellipticity implies solvability for adjoint" idea. We give a convenient definition of parametrix.

10. Definition. Let $G$ be a vector space of formal $E$ Fourier series invariant for $P$ (i.e., $P G \subset G$ ). A map $Q: G \rightarrow G$ is called a parametrix for $P$ on $G$ if 
$P Q$ and $Q P$ are the identity on $G$ (except possibly for a finite dimensional subspace). If $F_{j} \subset G$ (all $j$ ), then $Q$ is an E-parametrix for $P$ on $G$ if, in addition, $G\left(F_{j}\right) \subset F_{j}$ for all $j$.

11. Theorem. Let $P$ be an E-invariant differential operator. The following are equivalent:

(a) $P$ is $(G H)$.

(b) $P$ bas a parametrix on $C^{\infty}(M)$.

(c) $P$ bas an E-parametrix on $C^{\infty}(M)$.

(d) $P$ bas a parametrix on $\mathfrak{D}^{\prime}(M)$.

(e) $P$ has an E-parametrix on $\mathscr{D}^{\prime}(M)$.

Proof. Since $P F_{j} \subset F_{j},(\mathrm{~b})$ and (c) (respectively, (d) and (e)) are equivalent. If there exists a parametrix $Q$ on $\mathscr{D}^{\prime}(M)$ or $C^{\infty}(M)$, then $Q\left(F_{j}\right) \subset F_{j} \quad(j$ large), and $\hat{Q}(j)$ (Q restricted to $\left.F_{j}\right)$ must be $(\hat{P}(j))^{-1}$, for $j$ large. Suppose $(\mathrm{GH})$ is false. We construct a sequence $\left\{f_{a}\right\}$ as in Theorem 6. Put $g_{N}=\Sigma_{a>N} f_{a}$. $g_{N} \in \mathfrak{D}^{\prime}(M)-C^{\infty}(M)$, and $P g_{N} \in C^{\infty}(M)$. Thus $P$ can have no parametrix on $C^{\infty}(M)$. $P$ has no parametrix on $\mathcal{D}^{\prime}(M)$, for $P T=g_{N}$ has no solution in $\mathcal{D}^{\prime}(M)$. If $(\mathrm{GH})$ is true, it is easy to construct $Q$ as desired.

The estimate (LM) also suffices to insure the continuity of parametrices for $P$. In suitable cases, Rellich's lemma applies to give compactness.

12. Theorem. Suppose $P$ is $(G H)$. Then the parametrices for $P$ are continuous. If $b(P)>0$, the parametrices for $P$ on $C^{\infty}(M)$ are compact.

13. Theorem. Suppose $P$ is $(G H)$. Then $P: C^{\infty}(M) \rightarrow C^{\infty}(M)$ bas closed range, and is Fredholm with index 0.

This is true since $P$ and $P^{*}$ are both $(\mathrm{GH})$, and $\hat{P}(j)$ is invertible for $j$ large.

We define a different type of hypoellipticity.

14. Definition. $P$ is globally analytic hypoelliptic $(\mathrm{GAH})$ iff whenever $P f=$ $g, f \in \mathscr{D}^{\prime}(M), g \in A(M)$, then $f \in A(M)$.

15. Remarks. We could substitute $A^{\prime}(M)$ for $\mathscr{D}^{\prime}(M)$ in Definition 14. There would be no difference in the results to follow. We note that Seeley proves [9] that if $P$ is an $E$-invariant operator, with $E$ real analytic, then $P(A(M)) \subseteq A(M)$.

16. Theorem. $P$ is $(G A H)$ if and only if for any positive number $K$, there is a positive integer $N_{K}$ so that

$$
m(\hat{P}(j)) \geq \exp \left(-K\left|\lambda_{j}\right|^{1 / e}\right), \quad \text { for } j \geq N_{K}
$$

Proof. Use Theorem 1.6 as Theorem 1.5 was used in the proof of Theorem 6 . 
17. Theorem. If $P$ is $(G H)$, then $P$ is $(G A H)$.

Proof. (LM) implies (KN).

18. Remark. The converse is not true (see [2]).

Theorems $9,11,12$, and 13 remain true if for $(\mathrm{GH}), C^{\infty}(M)$, and $\mathcal{D}^{\prime}(M)$ we substitute $(\mathrm{GAH}), A(M)$, and $A^{\prime}(M)$. The proofs would use $(\mathrm{KN})$ as (LM) was used. The real-analytic version of Theorem 11 thus implies, in particular, that even if $m(\hat{P}(j)) \neq 0$ for $j$ large, $P$ is not (approximately) invertible in $A^{\prime}(M)$ when $(\mathrm{KN})$ is false. That is, if $(\mathrm{KN})$ is false, $P$ is not solvable even in the space of analytic functionals, even though it is solvable on finite sums of eigenfunctions. (An example of such $P$ is given in [2].)

It is clear now that any simple " $E$ " growth condition $\left(C^{\infty}, A\right)$ will give equally simple " $E$ " global hypoellipticity and solvability criteria (LM, KN).

3. Applications to compact homogenous spaces. Let $G$ be a compact Lie group and let $K$ be a closed connected subgroup of $G$. Let $\langle$,$\rangle be a G$-invariant Riemannian structure on $G / K=M$ derived from a bi-invariant one on $G$. Let $\Delta$ be the Laplace-Beltrami operator of $(M,\langle\rangle$,$) . The Peter-Weyl theorem combined$ with Frobenious reciprocity (see e. g. [11]) implies that

$$
L^{2}(M)=\sum_{\gamma \in \hat{\hat{G}}} V_{\gamma} .
$$

Here $\hat{G}$ is the set of all equivalence classes of irreducible unitary (hence finite dimensional) representations of $G . V_{\gamma}$ is a closed subspace if $L^{2}(M)$ such that if $W$ is an irreducible subspace of $V_{\gamma}$ then the action of $G$ on $W$ is in the class $\gamma$. Furthermore, $V_{\boldsymbol{\gamma}}$ is equivalent to the sum of $m_{\gamma}$ copies of $\gamma$ where $m_{\gamma}$ is computed as follows: let $\chi_{y}$ be the character of $\gamma$. Then

$$
m_{\gamma}=\int_{K} \chi_{\gamma}(k) d k
$$

(Here $d k$ will denote Haar measure on $K, d g$ Haar measure on G.) The projection $P_{\boldsymbol{y}}: L^{2}(M) \rightarrow V_{\boldsymbol{y}}$ is

$$
P_{\gamma} f(X)=\int_{G} d(\gamma) \overline{\chi_{\gamma}}(g) f\left(g^{-1} x\right) d g
$$

As is well known $\Delta \circ P_{y}=P_{y} \circ \Delta$. Hence $\Delta \mid V_{y}=\lambda_{\gamma} I$. Let now $X$ be in the Lie algebra of $G$, \&. Then $X$ induces a vector field $X^{*}$ on $M$ by

$$
\left(X^{*} f\right)(X)=\left.\frac{d}{d t} f(\exp (-t X) x)\right|_{t=0}
$$

Since $X^{*}$ is a Killing field $X^{*} \Delta=\Delta X^{*}$. Let $U(\&)$ be the complexified universal 
enveloping a lgebra of $\&$ (the left invariant differential operators on $G$ ). Then we have a map $D \mapsto D^{*}$ of $U(\&)$ into the differential operators on $M$. If $D$ is in $U(B)$ then $D^{*} \Delta=\Delta D^{*}$ since $D^{*}$ is just a linear combination of products of the $X^{*}$.

Let $T$ be a maximal torus of $G$ and let $P$ be a Weyl chamber of $T$. If $\gamma$ is in $\hat{G}$ let $\Lambda_{y}$ be its highest weight. Then since $\Lambda_{y} \in \hat{T}$ and $\hat{T}$ is a lattice we may put an inner product on $\hat{T}$ (indeed take a basis of $\hat{T}$ and call it orthonormal). Define, for $\gamma$ in $\hat{K},\|\gamma\|=\left\|\Lambda_{\gamma}\right\|$.

Lemma. There are constants $C_{1}, C_{2}$ so that

$$
C_{2}\|\gamma\|^{2} \leq\left|\lambda_{\gamma}\right| \leq C_{1}\|\gamma\|^{2}
$$

For a proof see [11].

Let $\left(\pi_{\gamma}, U_{y}\right)$ be an element of the class $\gamma \in \hat{G}$. Then $\pi_{y}$ is a unitary representation of $G$ and if $X$ is in $\&$ we define $\left.\pi_{\gamma}(X) \doteq(d / d t) \pi_{\gamma}(\exp t X)\right|_{t=0}$. We therefore find that $\pi_{\gamma}(D)$ makes sense for each $D$ in $U(\&)$. Define

$$
m_{\gamma}(D)=\inf _{\nu \in U_{\gamma}-\{0\}} \frac{\left\|\pi_{\gamma}(D) \nu\right\|}{\|\nu\|} .
$$

Then $m_{y}$ is independent of the choice of inner product on $\hat{T}$.

Theorem 6 now says

Theorem. Let $D$ be in $U(\&)$. Then $D^{*}$ is $(G H)$ if and only if there are constants $L, M$ ( $L$ positive) sucb that $m_{\gamma}(D) \geq L\|\gamma\|^{M}$ for all but a finite number of the $\gamma$ in $\hat{G}$ such that $V_{y} \neq(0)$.

Examples. 1. Let $G=S U(2)$. We take a basis $X_{1}, X_{2}, X_{3}$ of $\&$ so that $\left[X_{1}, X_{2}\right]=2 X_{3},\left[X_{1}, X_{3}\right]=-2 X_{2},\left[X_{2}, X_{3}\right]=2 X_{1}$. The representations of $G$ are indexed by nonnegative integers $k$. Take $\Delta=X_{1}^{2}+X_{2}^{2}+X_{3}^{2}$. Then $\pi_{k}(\Delta)=$ $-k(k+2)$. We can find a basis of $U^{k}$ (the representation space of $\pi_{k}$ ) so that

$$
\pi_{k}\left(X_{3}\right) \nu_{j}=i(k-2 j) \nu_{j}, \quad \pi_{k}\left(X_{2}\right) \nu_{j}=-j \nu_{j-1}+(k-j) \nu_{j+1}
$$

and

$$
\pi_{k}\left(X_{1}\right) \nu_{j}=-i\left(j \nu_{j-1}+(k-j) \nu_{j+1}\right) .
$$

Let $D=X_{1}^{2}+X_{2}^{2}=\Delta-X_{3}^{2}$. Then $\pi_{k}(D)$ is diagonalizable with eigenvalues

$$
-k(k+2)+(k-2 j)^{2}=-2 k-4 j k+4 j^{2}, \quad j=0, \ldots, k .
$$

Thus the smallest eigenvalue in norm is greater than or equal to $2 k$. Hence $m_{k}(D) \geq 2 k$. Thus $D$ is $(\mathrm{GH})$ and $b(D)=1$.

2. (Example 1 continued). Let $D=X_{1}^{2}+X_{2}^{2}-X_{3}^{2}$. This time $D=\Delta-2 X_{3}^{2}$ and has eigenvalues 


$$
-k(k+2)+2(k-2 j)^{2}=k^{2}-2 k-8 j k+8 j^{2}=\lambda(j), \quad j=0, \ldots, k,
$$

on $U^{k}$. Solving for $\lambda_{k}(j)=0$ we find that the solutions are

$$
j_{k}^{ \pm}=1 / 2(k \pm \sqrt{k(k+2) / 2}) \text {. }
$$

$j_{k}^{ \pm}$is an integer if and only if $\sqrt{k(k+2) / 2}$ is an integer. That is, if and only if $k(k+2)=2 m^{2}$ with $m$ an integer. This clearly implies that $j_{k}^{ \pm}$is an integer only if $k$ is even, say $k=2 r$. But then $m$ must be even, say, $m=2 s$. We find $r(r+1) / 2=s^{2}$. If $u$ is an integer such that $2 u^{2}+1=\nu^{2}$ then, setting $r=2 u^{2}$, $r(r+1) / 2=u^{2} \nu^{2}$. Hence if $k=4 u^{2}, j_{k}^{ \pm}=2 u^{2} \pm u \nu$, an integer. Now the equation $\nu^{2}-2 u^{2}=1$ is Pell's equation $\left(x^{2}-d y^{2}=1\right)$ with $d=2$, a nonsquare. Hence there are an infinite number of integral solutions to $\nu^{2}-2 u^{2}=1$ (see Landau [12, pp. 76-84]). This implies that $D=X_{1}^{2}+X_{2}^{2}-X_{3}^{2}$ is not globally hypoelliptic.

3 (Example 2 continued). Let $D=X_{1}^{2}+X_{2}^{2}-C X_{3}^{2}$ with $C$ an integer. Then on $U^{k}, D$ has eigenvalues

$$
-k(k+2)+(1+C)(k-2 j)^{2}=\lambda_{k}(j)
$$

Thus if $\lambda_{k}(j)=0$ we have $k(k+2)=(1+C)(k-2 j)^{2}$. Set $d=1+C ; m=k-2 j$. Then $k^{2}+2 k-d m^{2}=0$ or

$$
k=1 / 2\left(-2 \pm \sqrt{4+4 d m^{2}}\right)=-1 \pm \sqrt{1+d m^{2}} .
$$

Thus $\lambda_{k}(j)=0$ if and only if there is an integer $n$ so that $1+d m^{2}=n^{2}$. If $d$ is a square there is only a finite number of such $n, m$. Thus if $C=p^{2}-1$ with $p$ an integer there is only a finite number of $k$ such that $\lambda_{k}(j)=0$. Now $\lambda_{k}(j)$ is always an integer. Hence $\left|\lambda_{k}(j)\right| \geq 1$ for all but a finite number of $k, j$. This implies $D=X_{1}^{2}+X_{2}^{2}-\left(p^{2}-1\right) X_{3}^{2}$ is globally hypoelliptic for each integer $p$. (Note. for $p=1$ we have Example 1 but with a weaker estimate.)

Comparing this example with the analogous operators on $T^{3}$ (that is, let $\theta$, $\psi, \eta$ be mod 1 coordinates, $\left.X_{1} \leftrightarrow \partial / \partial \theta, X_{2} \leftrightarrow \partial / \partial \psi, X_{3} \leftrightarrow \partial / \partial \eta\right)$, then $\partial^{2} / \partial \theta^{2}$ $+\partial^{2} / \partial \psi^{2}-\left(p^{2}-1\right) \partial^{2} / \partial \eta^{2}$ is (GH) if and only if there are only a finite number of solutions to $k^{2}+l^{2}-\left(p^{2}-1\right) m^{2}=0$ ( $p$ an integer) $k, l, m$ integers. Setting $k=a m, l=b m$ we are left with solving $a^{2}+b^{2}-\left(p^{2}-1\right)=0$ or $a^{2}+b^{2}=p^{2}$ -1, for $a, b$ integers. There are many such $p$. For example, $p=1, p=3 \quad(a=2$, $b=2)$. Thus $\partial^{2} / \partial \theta^{2}+\partial^{2} / \partial \psi^{2}-\left(p^{2}-1\right) \partial^{2} / \partial \eta^{2}$ is not generally (GH) for $p$ an integer. (Note that on both $S U(2)$ and $T^{3}$ the operators considered above are not locally hypoelliptic when $p>1$. See $\$ 2$ of [6] for example.)

4. Let $D=X_{1}^{2}+X_{2}$. Then applying the result of Hörmander [6] we find that $D$ is (GH) (indeed locally hypoelliptic) and $b(D)=1$. This implies that there is a constant $C$ and $n \geq 0$ so that if $k \geq n$ then 
(*)

$$
\left\|\pi_{k}(D) \nu\right\| \geq C k\|\nu\|
$$

for all $\nu$ in $U^{k} .(*)$ is by no means obvious from the formulas for the action of B) on $U^{k}$.

5. Let $G$ be a compact Lie group. Let $\&$ be its Lie algebra and let $X_{1}$, $\cdots, X_{n}$ generate $\&$ as a Lie algebra. Applying Hörmander's theorem aga in we find that if $D=X_{1}^{2}+\cdots+X_{n-1}^{2}+X_{n}$ or $D=X_{1}^{2}+\cdots+X_{n}^{2}$ (cf. Example 1) there are constants $C_{1}, C_{2}$ such that for some $d>0$

$$
\left\|\pi_{\gamma}(D) \nu\right\| \geq C_{1}\|\gamma\|^{d}\|\nu\|
$$

for all $\nu$ in $V_{\gamma}$ and $\|\gamma\| \geq C_{2}$.

Note. In particular if $\&$ is semisimple, there exist $X_{1}, X_{2}$ generating $\&$.

Appendix. Subelliptic estimates. Let $M, d v$, and $E$ be as in $\S 1$.

Let $D$ be a differential operator of degree $d$ commuting with $E$. We decompose $f \in C^{\infty}(M)$ into its $E$-Fourier series: $f=\Sigma \hat{f}(j), f_{j} \in F_{j}$. Then $D f=$ $\Sigma \hat{D}(j) \hat{f}(j)$, and

$$
\|D f\|_{s}^{2}=\sum\left|\lambda_{j}\right|^{2 s / e}\|\hat{D}(j) \hat{f}(j)\|^{2} \geq \sum\left|\lambda_{j}\right|^{2 s / e} m(\hat{P}(j))^{2}\|\hat{f}(j)\|^{2} \bullet
$$

If $f \perp \operatorname{ker} D$, we see (when (LM) is valid):

$$
\|D f\|_{s}^{2} \geq L^{2} \sum\left|\lambda_{j}\right|^{2 s / e}\left|\lambda_{j}\right|^{2 M / e}\|\hat{f}(j)\|^{2} \geq L^{2}\|f\|_{s+M}^{2}
$$

when $M<b(D)$. We have shown the following result:

1. Proposition. Suppose $D$ commutes with $E$, and $D$ is $(G H)$. Then for any $m<b(D)$,

(a) $\|D f\|_{s} \geq C\|f\|_{s+t_{m}}$ when $f \perp$ ker $D$ in $H^{s}(M)$, and ker $D \subset C^{\infty}(M)$.

Remark. If $D$ is $E$-invariant with finite dimensional kernel $f \perp$ ker $D$ for one $H^{s}$ inner product implies perpendicularity for any $H^{s}$. In what follows we will not have $E$-invariance, and it is necessary to be explicit in our choice of perpendicularity.

2. Proposition. Let $D$ be any differential operator on $M$, and suppose $m>0$. Then $(\alpha)$ is equivalent to

$$
\|f\|_{s+m} \leq C\left(\|f\|_{s}+\|D f\|_{s}\right) \text { for } f \in C^{\infty}(M) .
$$

Proof. We first prove a lemma:

3. Lemma. If $D$ is a differential operator on $M$, and $\operatorname{ker} D \subset C^{\infty}(M)$, then $\operatorname{dim} \operatorname{ker} D<+\infty$.

Proof. If $d=\operatorname{deg} D$, then $D: H^{s}(M) \rightarrow H^{s-d}(M)$ is a continuous linear map. 
Put $K_{s}=\operatorname{ker} D \subset H^{s}(M) . K_{s}$ inherits a Hilbert space structure from $H^{s}(M)$. Let $i: H^{1}(M) \rightarrow H^{0}(M)$ be the usual injection. $i$ maps $K_{1}$ onto $K_{0}$ (since $\operatorname{ker} D C$ $C^{\infty}(M)$ ). But $i$ is compact (Rellich's lemma). So $\operatorname{ker} D=K_{1}=K_{0}$ is finite dimensional.

To prove $(\alpha) \Rightarrow(\beta)$ : If $f \in C^{\infty}(M)$, write $f=f_{1}+f_{2}$, where $f_{1} \in \operatorname{ker} D$, and $f_{2} \perp \operatorname{ker} D$ in $H^{s}(M)$. Since $\operatorname{dim} \operatorname{ker} D<\propto$, all norms on $\operatorname{ker} D$ are equivalent. Thus $\left\|f_{1}\right\|_{s+m} \leq C\left\|f_{1}\right\|_{s}$, for some $C$ independent of $f_{1}$. Using ( $\alpha$ ), we obtain

$$
\|f\|_{s+m} \leq\left\|f_{1}\right\|_{s+m}+\left\|f_{2}\right\|_{s+m} \leq C\left(\left\|f_{1}\right\|_{s}+\left\|D f_{2}\right\|_{s}\right) \leq C\left(\|f\|_{s}+\|D f\|_{s}\right) .
$$

$(\beta) \Rightarrow(\alpha)$ : If $(\beta)$ holds, and $f \in \mathscr{D}^{\prime}(M) \cap \operatorname{ker} D$, then $f \in H^{s}(M)$, for some $s$. $(\beta)$ immediately applies to show that $f \in S^{s+m}(M)$, etc. We continue, and obtain $f \in \cap H^{s}(M)=C^{\infty}(M)$. So $\operatorname{ker} D \subset D^{\infty}(M)$. To prove the inequality in ( $\left.\alpha\right)$, we assume that it is false and derive a contradiction. Thus we assume the existence of a sequence $\left\{f_{j}\right\} \subset C^{\infty}(M)$ so that $\left\|f_{j}\right\|_{s+m}=1,\left\|D f_{j}\right\|_{s} \rightarrow 0$, and $f_{j} \perp$ ker $D$ in $H^{s}(M)$. Rellich's lemma again applies to show that some subsequence (which we take again to be $\left.\left\{f_{j}\right\}\right)$ converges in $H^{s}(M): f_{j} \rightarrow g \in H^{s}(M)$. Then $D f_{j} \rightarrow D g$, but $\left\|D f_{j}\right\|_{s} \rightarrow 0$. So $g \in \operatorname{ker} D$. But $f_{j} \perp$ ker $D$ in $H^{s}(M)$, and $(\beta)$ applies to show that the set $\left\{\left\|f_{j}\right\|_{s}\right\}$ is bounded away from $0 . g \in\left\{\operatorname{ker} D \cap(\operatorname{ker} D)^{\perp}\right\}-\{0\}$, a contradiction.

3. Proposition. Let $D$ be any differential operator on $M$, and suppose $m>$ 0. Then $(\beta)$ implies

$$
\left\{\begin{array}{l}
\operatorname{ker} D \subset C^{\infty}(M), \text { and } D\left(C^{\infty}(M)\right) \text { is closed in } C^{\infty}(M) \text { with } \\
\text { the } \mathscr{D}^{\prime}(M) \text { relative topology (that is: suppose } D f_{j} \rightarrow g \\
\text { in some } \left.H^{S}(M), f_{j}, g \in C^{\infty}(M) . \text { Then } g \in \mathrm{im} D\right) .
\end{array}\right.
$$

Proof. Certainly ker $D \subset C^{\infty}(M)$ as in Proposition 2. The remainder of $(y)$ is a "classical" proof. We seek to show $g \in \mathrm{im} D$. We can assume each $f_{j} \perp$ ker $D$ in $H^{s}(M)$. First, suppose $\left\{\left\|f_{j}\right\|_{s}\right\}$ is bounded. Then $(\beta)$ combined with Rellich's lemma as before permits us to go to a subsequence (which we persist in calling $\left\{f_{j}\right\} \delta$ so that $f_{j} \rightarrow b$ in $H^{s}(M)$. Then $D b=g$ in $H^{s-d}(M)$. Since $m>0$ and $g \in C^{\infty}(M)$, it is easy to see that $b \in C^{\infty}(M)$.

If $\left\{\left\|f_{j}\right\|_{s}\right\}$ is unbounded, we pass to a subsequence (which we persist in calling $\left.\left\{f_{j}\right\}\right)$ so that $\left\|f_{j}\right\|_{s} \rightarrow \infty$. Consider $\tilde{f}_{j}=f_{j} /\left\|f_{j}\right\|_{s} \cdot D \tilde{f}_{j}=1 /\left\|f_{j}\right\|_{s} D f_{j} \rightarrow 0$ as $j=\infty$ (since $D f_{j} \rightarrow g$ in $H^{s}(M)$ ). Using the previous argument, we see that $\tilde{f}_{j} \rightarrow t \in H^{s}(M)$, and $D t=0$. But $\tilde{f}_{j} \perp$ ker $D$ in $H^{s}(M)$. So $t$ must be 0 . But $\|t\|_{s}=\lim _{j \rightarrow \infty}\left\|\tilde{f}_{j}\right\|_{s}=1$. This is a contradiction-the set $\left\{\left\|f_{j}\right\|_{s}\right\}$ must be bounded after normalization to be perpendicular to $\operatorname{ker} D$. 
4. Proposition. Let $D$ be any differential operator on M. Then $(\gamma)$ implies that $D$ is $(G H)$.

Proof. Suppose $D f=g, f \in \mathscr{D}^{\prime}(M), g \in C^{\infty}(M)$. Then $f \in H^{s}(M)$, for some s. We can find a sequence $\left\{f_{j}\right\} \subset C^{\infty}(M)$ so that $f_{j} \rightarrow f$ in $H^{s}(M)$. Then $D f_{j} \rightarrow D f$ in $H^{s-d}(M)$. $D f=g$ is in the closure (in $\left.C^{\infty}(M)\right)$ of $D\left(C^{\infty}(M)\right.$ ), in the $D^{\prime}(M)$ topology. Thus by $(y)$ there is $b \in C^{\infty}(M)$ with $D b=g$. But $D(f-b)=0$. So $f-b \in C^{\infty}(M)$, and $f \in C^{\infty}(M)$. Thus $D$ is $(\mathrm{GH})$.

5. Some comments on these conditions and their possible connections with hypoellipticity. We suspect that (GH) implies $(\gamma)$, and perhaps even that $(y)$ implies $(\alpha)$ for some $m \in R$. (Note that $(\beta)$ is vacuous for $m \leq 0$.) If $D$ is $E$-invariant, then it is easy to see that $(\alpha)$ is equivalent with condition (LM). In the examples we know of $(\mathrm{GH})$ vector fields, we have always found $(\alpha)$ holding, with $m$ negative.

We say $D$ is locally hypoelliptic (LH) if, for any open set $\Omega \subset M, D f=g$, $g \in C^{\infty}(\Omega), f \in \mathcal{D}^{\prime}(\Omega)$, then $f \in C^{\infty}(\Omega)$.

What additional conditions on an E-invariant operator besides (LM) imply (LH)?

If $\operatorname{deg} D=d$, then it is known that $(\beta)$ with $d-1<m \leq d$ implies that $D$ is (LH). See [10]. Even if $D$ is $E$-invariant, $(\beta)$ with $m=d-1$ does not imply (LH)-for there is the following example of C. Hoel: let $L$ be the constant coefficient third order operator on $R^{2}$ whose Fourier transform $\hat{L}(\xi, \eta)$ is $1+\xi^{2}+\eta^{2}$ $+i \xi \eta^{2}$. Since $L$ has constant coefficients, $L$ defines an operator on the 2 -torus, $T^{2}$, commuting with the constant coefficient Laplacian there. Because of the first three terms of $\hat{L}, L$ satisfies $(\beta)$ with $m=2$. So $L$ is $(\mathrm{GH})$. If $L$ were (LH) on $T^{2}$, then $L$ would be (LH) on $R^{2}$-but it is not (see Theorem 4.1.3 of [5]).

Note. It is well known that if $M=R^{n}$ and if $D$ is a constant coefficient operator of principal type $\left(D=p\left(\partial / \partial x_{1}, \cdots, \partial / \partial x_{n}\right), \sum_{j=1}^{n}\left|\partial p_{(\xi)} / \partial x_{j}\right|^{2} \neq 0\right.$ if $P(\xi)=0, \xi \neq 0)$, then $D$ satisfies a subelliptic estimate that looks like $(\beta)$ with $m=d-1$ where $d=\operatorname{deg} P$ (Hörmander [5, Theorem 3.3.7]). However, this subelliptic estimate is for compactly supported $C^{\infty}$ functions. It does not carry over to periodic functions (e.g., $\partial^{2} / \partial \theta^{2}-\partial^{2} / \partial \psi^{2}$ on $T^{2}$ is principle type but not (GH)).\}

This example shows that "complete continuity" of the associated Dirichlet norm is not enough. A very weak connection of (LH) with (GH) which we have not been able to prove (even in the $E$-invariant case) is: if the sup of the $m$ for which $(\alpha)$ holds is negative, then $D$ is not (LH). (So, for example, our vector fields would not be ( $\mathrm{LH}$ )-which is obvious.) 


\section{BIB LIOGRA PHY}

1. A. Cerezo and F. Rouvière, Solution élémentaire d'un opérateur différentiel linéaire invariant à gauche sur un groupe de Lie réel compact et sur un espace homogène réductif compact, Ann. Sci. Ecole Norm. Sup. (4) 2 (1969), 561-581. MR 42 \#6869.

2. S. Greenfield, Hypoelliptic vector fields and continued fractions, Proc. Amer. Math. Soc. 31 (1972), 115-118.

3. S. Greenfield and N. Wallach, Global hypoellipticity and Liouville numbers, Proc. Amer. Math. Soc. 31 (1972), 112-114.

4. - Globally hypoelliptic yector fields, Topology (to appear).

5. L. Hörmander, Linear partial differential operators, Die Grundlehren der math. Wissenschaften, Band 116, Academic Press, New York; Springer-Verlag, Berlin, 1963. MR 28 \#4221.

6. - Hypoelliptic second order differential equations, Acta Math. 119 (1967), 147-171. MR $36 \# 5526$.

7. L. Schwartz, Théorie des distributions. Tome I, Actualités Sci. Indust., no. 1091, Hermann, Paris, 1950. MR 12, 31.

8. R. T. Seeley, Integro-differential operators on vector bundles, Trans. Amer. Math. Soc. 117 (1965), 167-204. MR $30 \# 3387$.

9. - Eigenfunction expansions of analytic functions, Proc. Amer. Math. Soc. 21 (1969), 734-738. MR 39\#2180.

10. F. Treves, An invariant ciiterion of hypoellipticity, Amer. J. Math. 83 (1961), 645-668. MR 24\#A2732.

11. N. Wallach, Harmonic analysis on homogeneous spaces, Dekker, New York, 1973.

12. E. Landau, Elementare Zahlentheorie, Teubner, Leipzig, 1927; English transl., Chelsea, New York, 1958. MR 19, 1159.

DEPARTMENT OF MATHEMATICS, RUTGERS UNIVERSITY, NEW BRUNSWICK, NEW JERSEY 08903 